\title{
PROTOCOLOS Y MÉTODOS DE COLECTA PARA EL ESTUDIO DE ARTRÓPODOS DE DOSEL EN BOSQUES DE NIEBLA DEL NEOTRÓPICO
}

\section{PROTOCOLS AND METHODS TO STUDY OF CANOPY ARTHROPODS IN NEOTROPICAL CLOUD FOREST}

\author{
GASCA, A. HÉCTOR JAIME". M.Sc., HIGUERA, D. DIEGO'1. M.Sc. \\ ${ }^{1}$ Investigador. Corporación Sentido Natural. Colombia. \\ * Correspondencia: hjgasca@sentidonatural.org
}

Recibido: 12-01-2010; Aceptado: 23-06-2010

\section{Resumen}

Se presentan protocolos para la colecta y estudio de artrópodos del dosel de los bosques de niebla neotropicales. Para cada método se indican las ventajas y desventajas y se sugiere una estandarización de colecta para optimizar la investigación en el dosel. Se proporciona una clave para la elección de un método adecuado de colecta para investigadores interesados en el estudio de artrópodos de dosel en los bosques de niebla.

Palabras clave: dosel, artrópodos, métodos de colecta, bosque de niebla, Colombia

\begin{abstract}
Protocols and methods to collect and study canopy arthropods of neotropical cloud forests are presented. Advantages, disadvantages and collect standardization for each method are suggested, to optimize the research in the canopy. A key to choice an appropriate method of collection for researchers interested in the study of canopy arthropods in the cloud forest is provided.
\end{abstract}

Key words: canopy, arthropods, collection techniques, could forest, Colombia

\section{Introducción}

Los estudios enfocados en determinar la estructura de las comunidades de artrópodos residentes en el dosel de los bosques tropicales se ha incrementado rápidamente durante los últimos 20 años (STORK y BEST, 1994; STORK et al., 1997). Este estrato de los bosques húmedos tropicales contiene un porcentaje considerable de especies, siendo los artrópodos el grupo más diverso (STORK et al., 1997; BASSET et al., 2003A). El dosel de los bosques de regiones templadas y tropicales alberga pequeñas comunidades de artrópodos que contienen un gran número de especies aun sin describir, lo que ha ampliado mucho las estimaciones del número total de especies de insectos, y de artrópodos en general, concordando con las teorías que sostienen que las 
copas de los árboles proporcionan hábitats para una increíble diversidad (WILSON, 1988; ERWIN, 1988).

Una de las mejores formas de estudiar artrópodos es realizar excursiones científicas para la observación de sus hábitos y para colectarlos. Las colectas revelan información, a través de la observación directa, que muchas veces no está registrada en los libros o artículos científicos (RAFAEL, 2002). Los artrópodos de dosel presentan una gran diversidad debido a que poseen diferentes características biológicas en respuesta a la heterogeneidad ambiental que se genera en este estrato (SOUTHWOOD, 1978). La capacidad de los insectos para adaptarse a diferentes hábitats, sumado a los diferentes patrones de comportamiento, conllevan a la necesidad de establecer diferentes técnicas de colecta para el estudio de estos organismos. Debido a su abundancia y riqueza, sus variados hábitos y sus diferentes ciclos de vida, un solo método o técnica de colecta no es suficiente para el estudio de las comunidades de artrópodos que habitan el dosel de los bosques tropicales.

Uno de los métodos mas usados en el mundo para el estudio de los insectos de las copas de los árboles es la termonebulización o "fogging". Este método utiliza un insecticida no residual de baja toxicidad liberado por una máquina termonebulizadora, el cual se propaga en el ambiente a ser explorado. Los insectos sufren el efecto del insecticida y caen en bandejas colectoras de gran diámetro, suspendidas cerca de un metro del suelo (RAFAEL, 2002). El "fogging" es una técnica recomendable para el estudio de factores de las comunidades de artrópodos como grupos tróficos, biomasa, tamaño corporal, y patrones de abundancia de especies (STORK y HAMMOND, 1997). Sin embargo, puede ser inadecuado para el estudio de aspectos como comportamiento y actividad diaria, siendo ineficiente para la colecta grupos específicos de artrópodos como ácaros o insectos minadores de hojas.

A pesar de su importancia ecológica, el dosel de los bosques de niebla en el trópico es uno de los hábitats menos estudiados. Estos bosques representan uno de los ecosistemas que más presiones sufre por estar localizado generalmente donde se han establecido asentamientos humanos. Esta condición hace que los bosques de niebla junto con los bosques secos sean de los ecosistemas tropicales más amenazados en el mundo (BROWN y KAPPELLE, 2001). Dentro de algunos de los resultados que se han obtenido en estudios de dosel realizados en bosques de niebla en Colombia, se ha encontrado que en este estrato se alberga una alta cantidad de biomasa epifita (HIGUERA y MARTíNEZ, 2006a), que junto con los troncos en descomposición, proporcionan un hábitat ideal para el establecimiento de diferentes comunidades de artrópodos (GASCA e HIGUERA, 2008). Así mismo, el dosel de los boques de niebla, aporta una alta cantidad de materia 
orgánica a través de la caída de hojarasca, contribuyendo así al ciclo de nutrientes del bosque (HIGUERA y MARTÍNEZ, 2006b).

El objetivo del presente trabajo es el de proporcionar una serie de métodos y protocolos para el estudio de comunidades de artrópodos que se establecen en diferentes hábitats del dosel de bosques de niebla en el Neotrópico. La descripción de estos métodos se hace principalmente pensando en que pueden ser empleados para detectar la presencia de las especies y poder observar sus hábitos, teniendo en cuenta que el conocimiento previo del hábitat aumenta las posibilidades del éxito de captura. Algunos métodos no suministran datos directos acerca de la biología de las especies, por lo tanto se debe tener precaución si se quieren usar para obtener información del comportamiento, hábitos o relaciones con el medio físico y con otros organismos. Para cada método se indican las ventajas y desventajas y se sugiere una estandarización de colecta, que sirva de herramienta para aquellos investigadores interesados en el estudio de insectos de dosel.

\section{Métodos de colecta}

Selección de los árboles y técnica de ascenso por cuerda simple: Cualquier tipo de trabajo que se haga en la altura tiene unos riesgos y es por eso que estos riesgos deben aminorarse al máximo. En el caso de la escalada de árboles, se debe tener en cuenta que el no seguir unos protocolos de seguridad para hacer el ascenso puede poner en riesgo no sólo a la persona que esta ascendiendo, si no también a las personas que están próximas al árbol. Es por eso que se debe inspeccionar muy bien el árbol al que se desee ascender, y evaluar e identificar todos los riesgos potenciales antes de subir. Es importante que cuando se llegue a un ecosistema, en lo posible se conozcan las especies de árboles que conforman el dosel, ya que esta información es necesaria cuando se quiere saber que tan resistentes son las ramas del árbol o que profundas son las raíces.

Para poder acceder al dosel se emplea la técnica de cuerda simple (BASSET et al., 2003) en donde el ascenso se realiza por medio de una cuerda estática con la ayuda de dispositivos llamados ascensores o "jummars" y equipo especializado de escalada como arneses, mosquetones, cintas de seguridad, ochos, cordinos de seguridad entre otros.

Existen diferentes técnicas de ascenso a los árboles de manera segura y siguiendo los procedimientos de seguridad adecuados. Estas técnicas dependen de los objetivos del proyecto y del presupuesto que se disponga para adquisición de equipos. Se debe puntualizar que dado que el fin de este artículo es dar una visión general del protocolo de muestreo de artrópodos, sólo se describen de manera breve los equipos de ascenso y la función que 
cumplen en la técnica. Sin embargo, se recomienda consultar literatura especializada en el tema la cual dará una mayor información sobre los equipos y los procedimientos. Es importante aclarar que la adquisición de estos equipos debe hacerse en sitios especializados, ya sea en almacenes de equipos de escalar o de trabajos de arboricultura.

El equipo básico para la técnica de acenso con cuerda simple consta de una cuerda estática, preferiblemente que sea para arboricultura, la cual tiene un diámetro de $11 \mathrm{~mm}$ y un largo de al menos 40 metros. Se debe tener presente que el largo de la cuerda varia dependiendo de la altura de los árboles y del sitio de la copa a donde se quiere ascender. La cuerda es uno de los equipos más importantes de esta técnica, puesto que es por ésta por donde se va a ascender y descender. A estas cuerdas se les debe hacer un mantenimiento y se deben seguir ciertas normas para su cuidado, por lo que se recomienda leer estas especificaciones en los respectivos manuales para así disminuir el riesgo de trabajo en las alturas.

Otro de los equipos necesarios son las cintas de seguridad. Estas cintas permiten asegurarse al árbol cuando la persona se encuentra en la copa del árbol. Es muy importante asegurarse con cintas ya que esto permitirá mayor estabilidad y seguridad, y por lo tanto se podrá realizar con mayor eficiencia el trabajo de colecta. En general, lo que se hace para asegurarse a las ramas es pasar la cinta, previamente cerrada con un nudo, por encima de la rama. Luego uno de los extremos del círculo se pasa por dentro del otro, esto genera un bucle que se debe halar hasta que la cinta queda asegurada a la rama. Después de esto se debe poner un mosquetón que conecte el arnés del escalador con la cinta de seguridad (JEPSON, 2005).

Para poder ascender por la cuerda se hace necesario que el escalador previamente se coloque un arnés de cintura y hasta donde sea posible un arnés de pecho. Esto es importante ya que permitirá tener una posición más cómoda cuando el escalador se encuentre suspendido en el aire. Los arneses de cintura y de pecho se pueden conectar por medio de un cordino de seguridad, aunque algunos arneses tienen la posibilidad de conectarse con un mosquetón u óvalo de seguridad.

El equipo que va permitir el ascenso son los ascensores o "jummars". Para la técnica de cuerda simple es necesario dos ascensores, uno izquierdo y otro derecho. Estos son unos equipos con forma de mancorna que se colocan en la cuerda, tienen un movimiento ascendente, nunca descendente, permitiendo el ascenso. Estos ascensores se conectan por medio de cintas de seguridad a los pies, siendo la cinta un círculo cerrado, cuya longitud variara dependiendo de la altura de la persona. Es recomendable que uno de los círculos de cinta sobrepase la altura de la persona y que el otro quede al nivel de su cara. La 
decisión de cual cinta se deja más larga y cual más corta depende de si la persona es derecha o izquierda, el sistema ascensor cinta más largo va en el lado donde la persona tenga más manejo de su cuerpo y tenga más fuerza. Este sistema ascensor-cinta, que conecta los pies con las manos, por medio de un círculo de cinta para cada costado, pasa a través del cinturón del arnés de cintura y también atraviesa el sistema que asegura el arnés de cintura con el arnés de pecho.

Los equipos que se usan para el descenso en esta técnica corresponden a elementos diseñados para reducir y controlar la velocidad con que se baja. Se recomienda tener un dispositivo llamado "ATC" o un "ocho", cada uno con su respectivo mosquetón de seguridad. Un último equipo que sirve para controlar el ascenso y el descenso es el "shunt", dispositivo que va conectado al arnés a través de un círculo con cordino de seguridad, el cual se asegura con un nudo especial llamado llano riso.

Después de escoger el lugar en el dosel en donde se va a realizar la colecta, se lanza con una cauchera, un plomo atado a un nylon por la horqueta seleccionada. Cuando el nylon ha pasado por la horqueta y las dos puntas del nylon alcanzan el suelo, se procede a cambiar el nylon por una cuerda más fuerte, que puede ser una cuerda tejida de $3 \mathrm{~mm}$. Colocada esta cuerda por encima de la horqueta, y con las dos puntas en el suelo, se procede a subir la cuerda por donde se va ascender. Luego, uno de los extremos se amarra a la base del árbol, mientras que la otra punta se deja libre para empezar el ascenso.

Colocada la cuerda se procede a instalar los ascensores, ya conectados a los pies del escalador y sujetando cada ascensor con la mano correspondiente. Es importante colgarse por un par de minutos de la cuerda por donde se va a escalar para cerciorarse de que la rama es resistente. Colocados los equipos y verificado esto se procede al ascenso. El ascenso se hace por medio de una sincronización entre la pierna y el brazo del mismo lado en donde el escalador va ganando altura.

Para facilitar la colecta y observación de artrópodos en el dosel pueden ser construidas plataformas sobre las ramas gruesas de los árboles de estudio. Estas plataformas le permitirán al investigador tener mayor estabilidad a la hora de tener que manipular algún tipo de equipo para colectar las muestras deseadas. Además permitirán que el investigador pueda permanecer más tiempo en el dosel, ya que le dará la posibilidad de descansar. Otra de las ventajas que tienen las plataformas es que será más cómodo el descenso. Es recomendable que estas plataformas sean construidas en un sistema colgante y que no estén adheridas al árbol, para garantizar la plasticidad del sistema. 
Trampa aérea de intercepción de vuelo de dosel: La trampa de intercepción de vuelo para colectas de artrópodos en el dosel, es un modelo modificado de la trampa de tipo tienda Malaise, descrita por el himenopterólogo sueco René Malaise (MALAISE, 1937), y utilizada generalmente para colectar insectos voladores que habitan los estratos bajos del bosque, que presentan tendencia a subir cuando encuentran un obstáculo vertical. La trampa para el dosel tiene el mismo principio de captura y consiste en dos embudos hechos en tela de nylon de color blanco los cuales estaba unidos a dos rectángulos de tela de nylon negra cruzados perpendicularmente para formar las barreras centrales. Al extremo de cada embudo se ubican frascos de plástico colectores que contienen como sustancia letal alcohol al 70\% (Fig. 1). Algunos entomólogos utilizan un material llamado cinta piretroide, la cual contiene atrayentes letales para los insectos que ingresan al frasco colector. La trampa Malaise aérea es recomendable para colectas de artrópodos con una respuesta positiva a la luz los cuales se desplazan en un espacio tridimensional en el dosel (FINNAMORE et al., 2002).

Esta trampa es ideal para la captura de insectos voladores, principalmente pertenecientes a los órdenes Hymenoptera y Diptera. Pueden ser montadas por tiempo indeterminado, de día y de noche y a diferentes alturas en el dosel. Para una captura eficiente, es recomendable que la trampa sea instalada en las ramas altas del dosel superior, en áreas con espacios claros y abiertos entre el suelo y el dosel, orientando el frasco colector superior en el sentido de mayor luminosidad, y de preferencia en sentido transversal a la dirección del viento (RAFAEL, 2002).

El material colectado en los frascos colectores debe ser transferido a recipientes limpios con alcohol al $70 \%$, para luego ser llevados al laboratorio y realizar el proceso de separación e identificación. El contenido del frasco colector superior y el inferior será tomado como una misma muestra. El recipiente de la muestra final debe ser debidamente etiquetado con un número de identificación, la fecha y el lugar en el dosel en donde fue instalada la trampa.

La trampa suspensa, descrita por RAFAEL Y GORAYEB (1982), cumple el mismo principio de la trampa de intercepción de vuelo del dosel, pudiendo ser instalada a diferentes alturas. Sin embargo, tiene la desventaja de carecer del frasco colector inferior, reduciendo así la eficiencia de captura.

Colecta de muestras de liquen y musgo: La acumulación de los suelos suspendidos conformados por musgo, líquenes y acumulaciones de debris que se dispone de manera discontinua a lo largo de las altas ramas de los árboles en el dosel, es denominada suelos suspendidos. Los factores que responden a la abundancia y riqueza de artrópodos en este microhábitat, están directamente 
relacionados a la arquitectura del árbol que permite la acumulación de debris y subsecuentemente la formación de suelos suspendidos con el tiempo. La acumulación de este material en las ramas y en la corona varía entre especies de árboles y entre tipos de bosques. Los bosques altoandinos, y en especial, los bosques de roble acumulan una considerable cantidad de suelos suspendidos compuestos principalmente por musgo y liquen.

La artropofauna asociada a líquenes y musgo que se establece en el dosel superior puede ser examinada empleando la técnica de colecta manual de áreas de musgo y suelo (hand-held moss/soil corer). Esta técnica permite el muestreo de artrópodos de suelos suspendidos y tiene como principio tomar muestras de musgo y/o liquen de dosel de $3 \times 5 \mathrm{~cm}$, lo que corresponde a una o dos manotadas. También es posible definir un área de muestreo mayor, utilizando un rectángulo de aproximadamente $30 \times 10 \mathrm{~cm}$ o de $40 \times 20 \mathrm{~cm}$, el cual se coloca sobre la rama seleccionada para luego colectar el suelo suspendido que se encuentre dentro de esta área.

Para tomar las muestras en ocasiones es necesario usar un cuchillo para remover secciones de liquen que se encontraban muy adheridas al árbol. Cada muestra debe ser colocada dentro de una bolsa hermética sello-pack y posteriormente ser llevada a un embudo Berlese por un período de 48 horas para recolectar los artrópodos asociados a este microhábitat. El embudo Berlese, sistema ideado por el doctor Antonio Berlese (PASTRANA, 1985), se compone de un embudo al que en la parte inferior se adhiere un frasco con alcohol al $70 \%$. En la parte superior del embudo se coloca una tela metálica que recibe la muestra de musgo, hojarasca, etc. Como fuente de calor y luz se coloca una lámpara eléctrica en la parte superior, que calienta lentamente el material en observación. Los insectos que tienen fototropismo negativo, se desplazan hacia la parte inferior donde serán colectados en el frasco que contiene alcohol (Fig. 2). El método Berlese permite cuantificar el número de organismos que se hallan en los suelos suspendidos del dosel en el momento de la toma de la muestra.

Este método es ideal para la caracterización de las pequeñas comunidades de artrópodos que inician los procesos de descomposición de materia orgánica que se acumula en el dosel. 


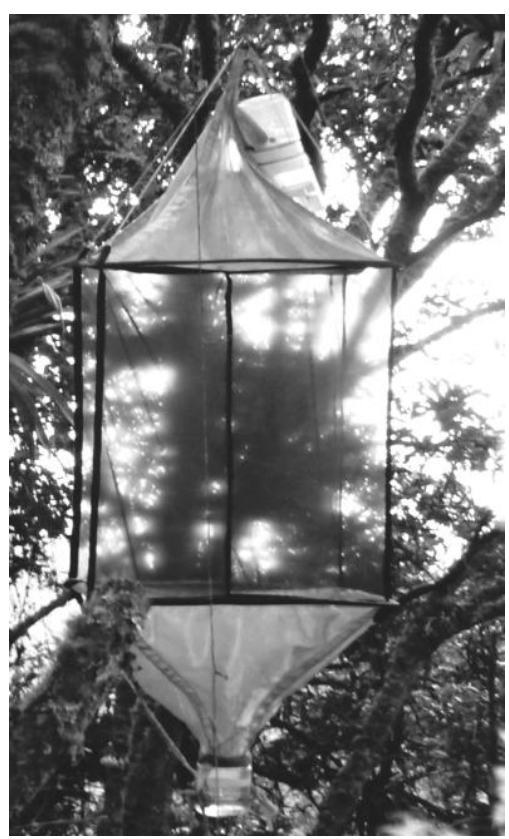

Figura 1. Trampa aérea de intercepción de vuelo

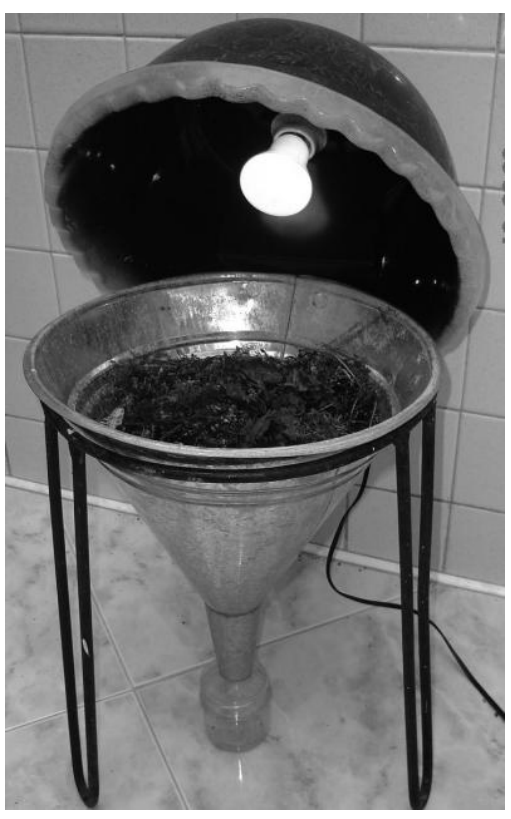

Figura 2. Embudo Berlese para muestras de suelos suspendidos de dosel.

Revisión de brómelias de dosel: La fitotelma de las brómelias, ambiente acuático con gran riqueza de organismos cuto recipiente es un vegetal (RINGUELET, 1962), contiene reservas de agua y acumulaciones de hojarasca y materia orgánica en descomposición durante todo el año por lo que pueden sostener complejas comunidades de artrópodos. Es recomendable colectar en brómelias de gran porte, tipo tanque, que están localizadas en los estratos correspondientes al dosel, preferiblemente en la corona externa de los árboles. Debido a la disposición de sus hojas y por sus mecanismos de captación e introducción de nutrientes, las brómelias tipo tanque tienen en su parte basal un embudo capaz de retener agua y materia orgánica, el cual es aprovechado por muchos artrópodos entre otros organismos como fuente de hábitat o de alimento (RINGUELET, 1962). La existencia de plataformas puede facilitar la colecta y manipulación de brómelias de gran tamaño.

Posteriormente cada planta debe ser colocada cuidadosamente dentro de una bolsa plástica para luego ser llevada al laboratorio. Los artrópodos asociados a cada individuo se recolectan deshojando poco a poco la planta y lavando las hojas con agua destilada. Para la caracterización de invertebrados, se recomienda filtrar el resultante con papel filtro de $80 \mathrm{~g}$ (OSPINA-BAUTISTA et al., 2004). Cada planta es considerada como una unidad de muestra y el material colectado debe ser depositado en un frasco con alcohol al 70\%. Así mismo, cada frasco debe estar debidamente etiquetado con el número de la muestra, identificación de la especie de bromólia, ubicación en el dosel y fecha de colecta. 
Algunos datos pueden ser útiles para realizar análisis que puedan relacionar las características de las plantas con la riqueza y abundancia de artrópodos asociados. El tamaño y capacidad de retención de hojarasca y materia orgánica de cada individuo pueden ser establecidos registrando algunas medidas morfométricas de las plantas como: 1) diámetro de la roseta, medido como la distancia entre las hojas más externas, 2) altura de la planta, 3) volumen de la roseta, calculado a partir del volumen de un cono, $V=\left(\pi r^{2} h\right) / 3$, donde $V$ es el volumen de la planta; y 4) el peso seco de la planta (OSPINABAUTISTA et al., 2004).

Este método es específico para comunidades de artrópodos residentes en plantas epifitas de dosel que por su arquitectura, acumulan materia orgánica en su interior, tales como las bromelias, las cuales pueden llegar a ser muy abundantes en los estratos superiores de los bosque de niebla del neotrópico, y en particular en bosques de roble.

Colecta y revisión de troncos en estado de descomposición: La madera en descomposición que se encuentra en el dosel y que aun no ha caído al suelo es uno de los microhábitat menos estudiados en ecología y caracterización de fauna asociada al dosel de bosques tropicales. La cantidad de troncos en descomposición que se pueden encontrar en el dosel, estimada en términos de biomasa, puede llegar a ser importante, convirtiéndose en un recurso alterno para insectos de hábitos saproxilófagos que encuentran un hábitat ideal para desarrollar funciones vitales (GASCA e HIGUERA, 2008). Sin embargo, en el dosel superior los troncos en descomposición representan un recurso relativamente discontinuo y discreto a lo largo de un transecto imaginario (BASSET et al., 2003B), lo que estaría limitando la capacidad de dispersión y establecimiento de comunidades de artrópodos en este microhábitat.

Para caracterizar las comunidades de artrópodos asociadas al microhábtat de la madera en descomposición, deben ser seleccionados troncos localizados en el dosel superior. La presencia de plataformas en el dosel, puede facilitar el proceso de observación, corte y colecta de los troncos. Luego se ser cortado, cada tronco debe ser colocado dentro de una bolsa plástica para luego en el suelo ser cortado cuidadosamente en pedazos con la ayuda de una hachuela, eliminando primero la corteza, en busca de la artropofauna asociada. Los artrópodos encontrados serán recolectados cuidadosamente empleando pinzas de punta fina y pinceles, para luego ser depositados en un frasco con alcohol al $70 \%$. Cada frasco debe estar etiquetado con el número de la muestra, la ubicación del tronco en el dosel y la fecha de colecta.

Para analizar relaciones entre la abundancia y riqueza de artrópodos con características físicas de los troncos, es recomendable pesar su biomasa, medir la longitud total y su diámetro, y anotar su estado de descomposición 
basándose en clasificación hecha por INGLES (1933) y seguida por FONSECA (1988) para troncos caídos en descomposición. Este ordenamiento presenta categorías de 1 a 5, siendo el estado 1 el comienzo de la descomposición, mientras que el estado 5 es el de mayor grado de descomposición. El estado 1 se establece después de la muerte del árbol, los hongos y bacterias comienzan a atacar la madera. La fauna inicial es de pequeños insectos que colonizan la corteza. En el estado 2 la corteza comienza a caerse. Termitas y otros insectos perforadores mayores (Coleópteros), comienzan a establecerse dentro del tronco. Es un estado con poca diversidad de artrópodos. El estado 3 se caracteriza por la presencia de galerías en el interior del tronco. Insectos considerados como grandes perforadores comienzan la verdadera colonización del tronco. La médula del tronco esta todavía fuerte mientras que el sub-cortex ya está colonizado por comunidades de artrópodos. La madera ya comienza a ablandarse. En el estado 4 el sub-cortex y la médula ya se encuentran completamente colonizados. Se establecen en este punto verdaderas comunidades de artrópodos establecidas, donde en algunos casos, insectos han colonizados galerías abandonadas por otros artrópodos. Finalmente en el estado 5 el tronco está completamente descompuesto. Varias comunidades de artrópodos ya se han retirado. La madera es esquelética y sin forma.

Este método de colecta es específico para el análisis de comunidades de insectos sapro-xilófagos o xilófagos estrictos que inician el proceso de descomposición de la madera en el dosel. Permite colectar no sólo individuos adultos, sino también individuos en estados larvales y de pupa, lo que puede ser útil para estudios sobre estructura de edades y fenología.

Clave para la orientación a investigadores usuarios de protocolos de muestreo en el dosel, para la elección de un método de colecta de artrópodos en bosques de niebla en el neotrópico (Adaptada de BASSET et al., 1997)

A continuación se presenta una clave dicotómica que sirve como guía para aquellas personas que estén interesadas en hacer estudios relacionados con muestreos, monitoreos e inventarios de biodiversidad de artrópodos asociados al dosel en bosques de niebla. La clave indica que método de colecta es el adecuado de acuerdo con el objetivo que el investigador persiga. 


\begin{tabular}{|c|c|c|}
\hline \multicolumn{3}{|c|}{$\begin{array}{c}\text { Clave dicotómica estudios relacionados con muestreos, monitoreos e } \\
\text { inventarios de biodiversidad de artrópodos asociados al dosel en } \\
\text { bosques de niebla. }\end{array}$} \\
\hline 1 & $\begin{array}{l}\text { Muestreos con énfasis en insectos } \\
\text { voladores con actividad diurna y nocturna }\end{array}$ & 2 \\
\hline 1 & $\begin{array}{l}\text { Muestreos con énfasis en colectas sobre } \\
\text { micro hábitats presentes en el dosel }\end{array}$ & 3 \\
\hline 2 & $\begin{array}{l}\text { Énfasis en insectos voladores con poca } \\
\text { actividad }\end{array}$ & $\begin{array}{l}\text { Jameos } \\
\text { desde plataformas } \\
\text { de dosel }\end{array}$ \\
\hline 2 & $\begin{array}{l}\text { Énfasis en grupos específicos de } \\
\text { Artrópodos asociados al estrato aéreo del } \\
\text { Como Coleoptera, Hymenoptera y Diptera }\end{array}$ & $\begin{array}{l}\text { Trampa aérea } \\
\text { Malaise de dosel }\end{array}$ \\
\hline 3 & $\begin{array}{l}\text { Énfasis en artrópodos descomponedores de } \\
\text { materia orgánica acumulada en el dosel y } \\
\text { con hábitos predadores }\end{array}$ & 4 \\
\hline 3 & $\begin{array}{l}\text { Énfasis en artrópodos con hábitos } \\
\text { herbívoros asociados al dosel de robledales }\end{array}$ & $\begin{array}{c}\text { Jameos } \\
\text { sucesivos } \\
\text { en el follaje }\end{array}$ \\
\hline 4 & $\begin{array}{l}\text { Énfasis en artropofauna asociada a epífitas } \\
\text { de dosel }\end{array}$ & 5 \\
\hline 4 & $\begin{array}{l}\text { Muestreos realizados en otro tipo de micro } \\
\text { hábitat presente en el dosel }\end{array}$ & 6 \\
\hline 5 & $\begin{array}{l}\text { Énfasis en fitotelmas y artropofauna } \\
\text { asociada a bromelias epífitas tipo tanque } \\
\text { ubicadas en el dosel }\end{array}$ & $\begin{array}{l}\text { Colecta de } \\
\text { bromelias } \\
\text { en el dosel }\end{array}$ \\
\hline 5 & $\begin{array}{l}\text { Énfasis en la colecta de artrópodos } \\
\text { asociados a epífitas de dosel con otro tipo } \\
\text { de arquitectura }\end{array}$ & $\begin{array}{l}\text { Colecta de bromelias } \\
\text { de pequeño tamaño, } \\
\text { ciperáceas, araceas, } \\
\text { epífitas. }\end{array}$ \\
\hline 6 & $\begin{array}{l}\text { Énfasis en artropofauna asociada a suelos } \\
\text { suspendidos en el dosel. Colecta de grupos } \\
\text { específicos como Colembola y Acarina }\end{array}$ & $\begin{array}{c}\text { Colecta de } \\
\text { musgo y liquen } \\
\text { (suelos suspendidos }\end{array}$ \\
\hline 6 & $\begin{array}{l}\text { Énfasis en artropofauna asociada a } \\
\text { madera en descomposición de roble } \\
\text { Colecta de grupos de artrópodos con } \\
\text { hábitos saproxilófagos }\end{array}$ & $\begin{array}{l}\text { Colecta de troncos en } \\
\text { descomposición } \\
\text { del dosel }\end{array}$ \\
\hline
\end{tabular}




\section{Agradecimientos}

Los autores agradecen a la Corporación Sentido Natural por su permanente apoyo. A Rufford Small Grants, Idea Wild y la Reserva Bosque Macanal por su apoyo financiero y logístico. Finalmente a Roberto Rodríguez por sus indicaciones recomendaciones para la construcción de plataformas en el dosel.

\section{Referencias}

BASSET, Y.; SPRINGATE, N.D.; ABERLENC, H.P.; DELVARE, G. 1997. A review of methods of collecting arthropods in tree canopies. Págs. 551-561 in: Stork, N.E.; Adis, J.; Didham, R.K. (eds.), Canopy Arthropods. Chapman and Hall. London

BASSET, Y.; NOVOTNY, V.; MILLER, S.E.; KITCHING, R.L. 2003a. Arthropods of Tropical Forests. Cambridge University Press. United Kingdom.

BASSET, Y.; HAMMOND, P.M.; BARRIOS, H.; HOLLOWAY, J.D.; MILLER, S.E. 2003b. Vertical stratification of arthropod assemblages. Págs. 474-543 in: Basset, Y.; Novotny, V.; Miller, S.E.; Kitching, R.L. (eds.), Arthropods of Tropical Forests. Cambridge University Press. United Kingdom.

BROWN A.; KAPPELLE, M. 2001. Introducción a los bosques del neotrópico: una síntesis regional. Instituto Nacional de Biodiversidad (INBio), Fundación Agroforestal del Noroeste de Argentina (FUA), World Conservation Union (IUCN), University of Amsterdam (IBED-UvA), Laboratorio de Investigaciones Ecológicas los Yungas de Argentina (LIEY). Editorial INBio. Santo Domingo de Heredia, Costa Rica.

ERWIN, T.L. 1988. The tropical forest canopy: the heart of biotic diversity. Págs. 123-129 in: E.O. Wilson, E.O (ed.), Biodiversity. National Academy Press, Washington D.C.

FINNAMORE, A.T.; WINCHESTER, N.N.; BEHAN-PELLETIER, V. M. Protocols for measuring biodiversity: Arthropod Monitoring in Terrestrial Ecosystems. [fecha de acceso 5 de enero de 2010]. URL Disponible: http://www.emanrese.ca/eman/ecotools/protocols/terrestrial/arthropods/a-malaise.html

FONSECA, C.R.V. 1988. Contribução ao conhecimento da bionomia de Passalus convexus Dalman, 1817 e Passalus latifrons Percheron, 1841 (Coleoptera: Passalidae). Acta Amazônica 18(1-2):197-222.

GASCA, H.J.; HIGUERA, D. 2008. Artrópodos asociados al dosel de un robledal de Quercus humboldtii Bonpl. (Fagaceae) de la reserva Bosque Macanal (Bojacá, Colombia). Boletín Sociedad Entomológica Aragonesa 43: 173-185. 
HIGUERA D.; MARTÍNEZ, E. 2006a. Sequestration and storage capacity of carbon in the canopy oak trees and their epiphytes in a Neotropic Cloud Forest, Colombia. Lyonia 11(1):17-23.

HIGUERA D.; MARTÍNEZ, E. 2006b. Litterfall and nutrient fluxes in canopy oaks in neotropical cloud forest, Colombia. Lyonia 11(1):67-74.

INGLES, L.G. 1933. The succession of Insects in Tree Trunks as Shown by the Collection from the Varius Stages of Decay. J. Ent. Zool. 25:57-59.

JEPSON, J. 2005. The tree climber's companion. A reference and training manual for professional trees climbers. 2nd edition. United States of America.

MALAISE, R. 1937. A new insect trap. Ent. Tidskr 58:148-160

PASTRANA, J.A. 1985. Caza, preparación y conservación de insectos. $2^{\text {da }}$ edición. Buenos Aires, Argentina.

OSPINA-BAUTISTA, F.; ESTEVES-VARÓN, J.; BETANCUR, J.; REALPEREBOLLEDO, E. 2004. Estructura y composición de macro invertebrados acuáticos asociados a Tillandsia turneri Baker (Bromeliaceae) en un bosque alto andino colombiano. Acta Zoológica Mexicana 20(1):153-166.

RAFAEL, J.A.; GORAYEB, I.S. 1982. Tabanidae (Diptera) da Amazônia I. Uma nova armadilha suspensa e primeiros registros de mutucas de copas de árvores na Amazônia. Acta Amazônica 12(1): 232-236.

RAFAEL, J.A. 2002. A amostragem. Protocolo e técnicas de captura de Diptera.Proyecto de Red Ibneroamericana de Biogeografia y Entomología Sistemática PrIBES 2002. Monografias Tercer Milenio. Vol. 2. Sociedad Entomológica Aragonesa (SEA) and Cyted, Zaragoza.

RINGUELET, R.A. 1962. Ecología Acuática Continental. Editorial Universitaria de Bs. As.

SOUTHWOOD, T.R.E. 1978. Ecological methods with particular reference to the study of insect populations. $2^{\text {da }}$ edition. Chapman and Hall, London.

STORK, N.E.; BEST, V. 1994. European Science Foundation - results of a survey of European canopy research in the tropics. Selbyana 15:51-62.

STORK, N.E.; HAMMOND, P.M. 1997. Sampling arthropods from tree-crowns by fogging with knockdown insecticides: lessons from studies of oak tree beetle assemblages in Richmond Park (UK). Págs. 3-26. in: Stork, N.E.; Adis, J.A.; Didham, R.K. (eds.), Canopy Arthropods. Chapman and Hall, London.

STORK, N.E.; ADIS, J.; DIDHAM, R.K. (eds.) 1997. Canopy Arthropods. Chapman and Hall, London. 
WILSON E.O.M. 1988. The current state of biological diversity. Pp. 3-17 In: E.O.M. 